\title{
VOLUME XXIX
}

\section{INDEX OF PAPERS BY AUTHORS}

Author

ABDELMALEK, NABIH N.

AHLBERG, J. H. \& ITO, T.

ANDERSON, NED

APOSTOL, TOM M.

ATKINSON, M. D.

BAKER, GARTH A.

BARNHILL, R. E. \& GREGORY, J. A.

BARWELL, V. K.

BATEMAN, PAUL T., PURDY, GEORGE B. \& WAGSTAFF, SAMUEL S., JR.

BEAM, RICHARD M.

BERLEKAMP, ELWYN R. BLEICHER, M. N. \& ERDÖS, P. BOROSH, I., MORENO, C. J.

\& PORTA, $\mathrm{H}$.

BOULLION, THOMAS

BRAMBLE, JAMES H., NITSCHE, JOACHIM A. \& SCHATZ, ALFRED H. BREMNER, ANDREW BRENT, RICHARD P.

BRILLHART, JOHN, LEHMER, D. H. \& SELFRIDGE, J. L. BRILLHART, JOHN

BRILLHART, JOHN BRODLIE, KENNETH W.

BURR, STEFAN A.

CARASSO, ALFRED

CARLITZ, L. \& SCOVILLE, RICHARD

CARTA, DAVID G.
Title

Page

An Efficient Method for the Discrete Linear $L_{1}$ Approximation Problem

A Collocation Method for Two-Point Boundary Value Problems

A Generalization of the Method of Averages for Overdetermined Linear Systems

The Resultant of the Cyclotomic Polynomials $F_{m}(a x)$ and $F_{n}(b x)$

An Algorithm for Finding the Blocks of a Permutation Group

A Finite Element Method for First Order Hyperbolic Equations

Polynomial Interpolation to Boundary Data on Triangles ..

See: HEADLEY, V. B. \& BARWELL, V. K. ....................... 863

726

Some Numerical Results on Fekete Polynomials .................

See: WARMING, R. F., BEAM, RICHARD M. \& HYETT, B. J.

The Design of Slowly Shrinking Labelled Squares ............... 25

The Number of Distinct Subsums of $\Sigma_{1}^{N} 1 / i \ldots \ldots \ldots \ldots \ldots \ldots . . . . . . . . . .29$

Elliptic Curves Over Finite Fields. II ................................... 951

See: SINGH, INDERJIT, POOLE, GEORGE \& BOULLION, THOMAS

Maximum-Norm Interior Estimates for Ritz-Galerkin Methods

An Equation of Mordell

Irregularities in the Distribution of Primes and Twin Primes

See: HALLYBURTON, JOHN C., JR. \& BRILLHART, JOHN

See: MORRISON, MICHAEL A. \& BRILLHART, JOHN .. 183

On Bairstow's Method for the Solution of Polynomial Equations

On Detecting a Periodic Event by Means of Periodic Observations. I

Coupled Sound and Heat Flow and the Method of Least Squares

A Generating Function for Triangular Partitions

Low-Order Approximations for the Normal Probability Integral and the Error Function 
CIMENT, MELVYN \& LEVENTHAL, STEPHEN H.

COHEN, FRED \& SELFRIDGE, J. L.

COOK, GRANT

DIETER, U.

DOUGLAS, JIM, JR. \& DUPONT, TODD

DOUGLAS, JIM, JR. \& DUPONT, TODD

DOUGLAS, JIM, JR., DUPONT, TODD \& WAHLBIN, LARS

DUNHAM, CHARLES B.

DUNHAM, CHARLES B.

DUNHAM, CHARLES B.

DUPONT, TODD

DUPONT, TODD

DUPONT, TODD

EHLE, BYRON L. \& PICEL, ZDENEK

ERDÖS, P.

ERDÖS, P., GRAHAM, R. L., RUZSA, I. Z. \& STRAUS, E. G.

ESPER, N.

FERGUSON, HELAMAN ROLFE PRATT, NIELSEN,

DALE E. \& COOK, GRANT

FIELDS, JERRY L. \& ISMAIL, MOURAD E. $\mathrm{H}$.

FORNBERG, B. \& KÖLBIG, K. S.

GAUTSCHI, WALTER

GERTH, FRANK III

GILL, PHILIP E., MURRAY, WALTER \& SAUNDERS, MICHAEL A.

GRAHAM, R. L

GREGORY, J. A. GROETSCH, C. W.

GUPTA, G. K. \& WALLACE, C. S.

GUSTAFSSON, BERTIL

GUY, RICHARD K. \& SELFRIDGE, J. L.
Higher-Order Compact Implicit Schemes for the Wave Equation 985

Not Every Number is the Sum or Difference of Two Prime Powers

See: FERGUSON, HELAMAN ROLFE PRATT, NIELSEN, DALE E. \& COOK, GRANT

How to Calculate Shortest Vectors in a Lattice 827

The Effect of Interpolating the Coefficients in Nonlinear Parabolic Galerkin Procedures 360

A Galerkin Method for a Nonlinear Dirichlet Problem 689

Optimal $L_{\infty}$ Error Estimates for Galerkin Approximations to Solutions of Two-Point Boundarý Value Problems ..... 475

Chebyshev Approximation by Interpolating Rationals on $[\alpha, \infty)$

A Difficulty in Williams' Algorithm for Interpolating Rationals

Convergence of the Fraser-Hart Algorithm for Rational Chebyshev Approximation

See: DOUGLAS, JIM, JR. \& DUPONT, TODD 360

See: DOUGLAS, JIM, JR. \& DUPONT, TODD 689

See: DOUGLAS, JIM, JR., DUPONT, TODD \& WAHLBIN, LARS

Two-Parameter, Arbitrary Order, Exponential Approximations for Stiff Equations

See: BLEICHER, M. N.\& ERDÖS, P. .................................... 29

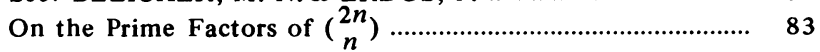

Tables of Reductions of Symmetrized Inner Products ("Inner Plethysms") of Ordinary Irreducible Representations of Symmetric Groups

A Partition Formula for the Integer Coefficients of the Theta Function Nome 851

Polynomial Expansions 894

Complex Zeros of the Jonquière or Polylogarithm Function 582

Nonexistence of Chebyshev-Type Quadratures on Infinite Intervals

A Note on $l$-Class Groups of Number Fields ......................... 1135

Methods for Computing and Modifying the $L D V$ Factors of a Matrix

See: ERDÖS, P., GRAHAM, R. L., RUZSA, I. Z. \& STRAUS, E. G.

See: BARNHILL, R. E. \& GREGORY, J. A. ....................... 726

On Existence Criteria and Approximation Procedures for Integral Equations of the First Kind 1105

Some New Multistep Methods for Solving Ordinary Differential Equations 489

The Convergence Rate for Difference Approximations to Mixed Initial Boundary Value Problems 396 What Drives an Aliquot Sequence? 
HABER, SEYMOUR

HAEGEMANS, ANN

HAGER, WILLIAM W. \& STRANG, GILBERT

HAGIS, PETER, JR. \& MCDANIEL, WAYNE L.

HALLYBURTON, JOHN C., J \& BRILLHART, JOHN

HAYES, JOHN K., KAHANER, DAVID K. \& KELLNER, RICHARD G.

HEADLEY, V. B. \& BARWELL, V. K.

HEDSTROM, G. W.

HENDY, M. D.

HERTLING, JÖRG \& SCHIOP, ALEXANDRU I.

HILL, DAVID R.

HUNKINS, DALTON R. HUNTER, D. B.

HYETT, B. J.

IKEBE, YASUHIKO

ISMAIL, MOURAD E. H. ITO, T.

JOHNSON, CLAES \& THOMÉE, VIDAR JOHNSON, WELLS KAHANER, DAVID K.

KELLER, H. B.

KELLNER, RICHARD G.

KEMP, MARILYN J.

KL $\varnothing$ VE, TORLEIV

KNUTH, DONALD E.

KÖLBIG, K. S.

LAKEIN, RICHARD B.

LEHMER, D. H.

LELAND, KENNETH O.

LEVENTHAL, STEPHEN H. LEWIS, JAMES T.

LIVNE, AVISHAI

LUKE, YUDELL L., TING, BING YUAN \& KEMP, MARILYN J. MCDANIEL, WAYNE L.
Adaptive Integration and Improper Integrals ......................... 806 See: PIESSENS, ROBERT \& HAEGEMANS, ANN ............ 810 Free Boundaries and Finite Elements in One Dimension .... 1020

On the Largest Prime Divisor of an Odd Perfect Number.II ............................................................................ 922

Two New Factors of Fermat Numbers ..................................... 109

A Numerical Comparison of Integral Equations of the First and Second Kind for Conformal Mapping

On the Distribution of the Zeros of Generalized Airy Functions

Models of Difference Schemes for $u_{t}+u_{x}=0$ by Partial Differential Equations

The Distribution of Ideal Class Numbers of Real Quadratic Fields

On the Stability of the Ritz-Galerkin Method for Hammerstein Equations

On Comparing Adams and Natural Spline Multistep Formulas

Cubatures of Precison $2 k$ and $2 k+1$ for Hyperrectangles ..1098

Some Properties of Orthogonal Polynomials 559

See: WARMING, R. F., BEAM, RICHARD M. \& HYETT, B. J.

The Zeros of Regular Coulomb Wave Functions and of Their Derivatives

See: FIELDS, JERRY L. \& ISMAIL, MOURAD E. H. ........ 894

See: AHLBERG, J. H. \& ITO, T. .......................................... 761

Error Estimates for a Finite Element Approximation of a Minimal Surface

Irregular Primes and Cyclotomic Invariants ........................... 113

See: HAYES, JOHN K., KAHANER, DAVID K. \& KELLNER, RICHARD G.

Approximation Methods for Nonlinear Problems with Application to Two-Point Boundary Value Problems

See: HAYES, JOHN K., KAHANER, DAVID K. \& KELLNER, RICHARD G.

See: LUKE, YUDELL L., TING, BING YUAN \& KEMP, MARILYN J.

Sums of Distinct Elements from a Fixed Set .......................... 1144

Estimating the Efficiency of Backtrack Programs .................. 121

See: FORNBERG, B. \& KÖLBIG, K. S. .............................. 582

Computation of the Ideal Class Group of Certain Complex Quartic Fields. II

See: BRILLHART, JOHN, LEHMER, D. H. \& SELFRIDGE, J. L.

An Algorithm for Winding Numbers for Closed Polygonal Paths

See: CIMENT, MELVYN \& LEVENTHAL, STEPHEN H. .. 985

Restricted Range Approximation and Its Application to Digital Filter Design

Seven-Point Difference Schemes for Hyperbolic Equations

On Generalized Gaussian Quadrature 1083 
McGUIRE, G. R. \& MORRIS, J. Ll.

MAHLER, K.

MARDER, B. M. MILLER, J. C. P. MILLS, W. H. MORENO, C. J. MORGAN, JOHN \& SCOTT, RIDGWAY

MORRIS, J. Ll.

MORRISON, MICHAEL A.

MORRISON, MICHAEL A. \& BRILLHART, JOHN MURRAY, WALTER

NEWBERY, A. C. R. NEWMAN, MORRIS NIELSEN, DALE E.

NITSCHE, JOACHIM A.

OLIVER, J.

OSBORN, JOHN E. OSLER, THOMAS J.

PANDA, REKHA

PANDIKOW, W. PENNEY, DAVID E. \& POMERANCE, CARL PICEL, ZDENEK PIESSENS, ROBERT \& HAEGE MANS, ANN POMERANCE, CARL

POMERANCE, CARL POOLE, GEORGE D. POOLE, GEORGE

PORTA, H.

PURDY, GEORGE B.

RAKOTCH, E.

RIORDAN, JOHN

ROBINSON, RAPHAEL M. ROSENBERG, IVO G. \&

STENGER, FRANK

ROSENBLATT, M.

ROSSER, J. BARKLEY \& SCHOENFELD, LOWELL ROTHSCHILD, LINDA PREISS RUSSELL, R. D. \&

VARAH, J. M. RUZSA, I. Z.

SAUNDERS, MICHAEL A.
Explicit-Implicit Schemes for the Numerical Solution of Nonlinear Hyperbolic Systems ……................................ 407

A Necessary and Sufficient Condition for Transcendency ... 145

GAP-A PIC-Type Fluid Code .............................................. 434

On Factorisation, with a Suggested New Approach .............. 155

Continued Fractions and Linear Recurrences ........................ 173

See: BOROSH, I., MORENO, C. J. \& PORTA, H. ............... 951

A Nodal Basis for $C^{1}$ Piecewise Polynomials of Degree

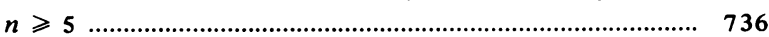

See: McGUIRE, G. R. \& MORRIS, J. Ll. .......................... 407

A Note on Primality Testing Using Lucas Sequences ............ 181

A Method of Factoring and the Factorization of $F_{7} \ldots \ldots \ldots . .183$

See: GILL, PHILIP E., MURRAY, WALTER \& SAUNDERS,

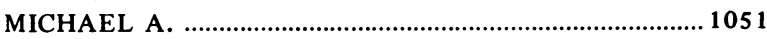

Polynomial Evaluation Schemes .............................................. 1046

Congruence Subgroups of the Modular Group ....................... 207

See: FERGUSON, HELAMAN ROLFE PRATT, NIELSEN, DALE E. \& COOK, GRANT …....................................... 851

See: BRAMBLE, JAMES H., NITSCHE, JOACHIM A. \& SCHATZ, ALFRED H. 677

A Curiosity of Low-Order Explicit Runge-Kutta Methods ... 1032

Spectral Approximation for Compact Operators ................... 712

An Identity for Simplifying Certain Generalized Hypergeometric Functions ............................................................. 888

See: SRIVASTAVA, H. M. \& PANDA, REKHA .................. 1115

See: ZAJTA, A. J. \& PANDIKOW, W. .................................. 566

Three Elliptic Curves with Rank at Least Seven ..................... 965

See: EHLE, BYRON L. \& PICEL, ZDENEK ....................... 501

Cubature Formulas of Degree Nine for Symmetric Planar Regions 810

The Second Largest Prime Factor of an Odd Perfect Number

See: PENNEY, DAVID E. \& POMERANCE, CARL ...............965

Generalized $M$-Matrices and Applications ……....................... 903

See: SINGH, INDERJIT, POOLE, GEORGE \& BOULLION, THOMAS 615

See: BOROSH, I., MORENO, C. J. \& PORTA, H. 951

See: BATEMAN, PAUL T., PURDY, GEORGE B. \& WAGSTAFF, SAMUEL S., JR.

Numerical Solution for Eigenvalues and Eigenfunctions of a Hermitian Kernel and an Error Estimate

The Distribution of Crossings of Chords Joining Pairs of $2 n$ Points on a Circle

The Structure of Certain Triple Systems ................................ 223

A Lower Bound on the Angles of Triangles Constructed by Bisecting the Longest Side 390

Multiply Schemes and Shuffling ........................................... 929

Sharper Bounds for the Chebyshev Functions $\theta(x)$ and

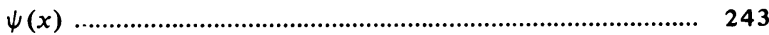

See: WANG, PAUL S. \& ROTHSCHILD, LINDA PREISS .. 935

A Comparison of Global Methods for Linear Two-Point Boundary Value Problems 1007

See: ERDÖS, P., GRAHAM, R. L., RUZSA, I. Z. \& STRAUS, E. G.

See: GILL, PHILIP E., MURRAY, WALTER \& SAUNDERS, MICHAEL A. 
Author

SCHATZ, ALFRED H.

ŞCHIOP, ALEXANDRU I. SCHOENFELD, LOWELL

SCOTT, RIDGWAY

SCOVILLE, RICHARD

SELFRIDGE, J. L.

SELFRIDGE, J. L.

SELFRIDGE, J. L.

SERBIN, STEVEN M.

SHANKS, DANIEL

SHAPIRO, RALPH

SINGH, INDERJIT, POOLE, GEORGE \& BOULLION, THOMAS

SKÖLLERMO, GUNILLA

SRIVASTAVA, H. M. \& PANDA, REKHA

ST ARK, H. M.

STENGER, FRANK

STEWART, G. W.

STRANG, GILBERT

STRAUS, E. G.

STRAUS, E. G.

TEMME, N. M.

THOMÉE, VIDAR

TING, BING YUAN

TUNG, S. H.

TURÁN, PAUL

VARAH, J. M.

WAGSTAFF, SAMUEL S., JR.

WAGSTAFF, SAMUEL S., JR.

WAHLBIN, LARS

WAHLBIN, LARS

WALLACE, C. S.

WANG, PAUL S. \& ROTHSCHILD, LINDA PREISS

WARMING, R. F., BEAM,

RICHARD M. \&

HYETT, B. J.

WEINBERGER, PETER J.

WEISS, RICHARD

WIMP, JET
Title

Page

See: BRAMBLE, JAMES H., NITSCHE, JOACHIM A. \& SCHATZ, ALFRED H. 677

See: HERTLING, JÖRG \& ŞCHIOP, ALEXANDRU I. .......... 484

See: ROSSER, J. BARKLEY \& SCHOENFELD, LOWELL .. 243

See: MORGAN, JOHN \& SCOTT, RIDGWAY …………….... 736

See: CARLITZ, L. \& SCOVILLE, RICHARD ……………..... 67

See: BRILLHART, JOHN, LEHMER, D. H. \& SELF-

RIDGE, J. L. .............................................................. 620

See: COHEN, FRED \& SELFRIDGE, J. L. ........................... 79

See: GUY, RICHARD K. \& SELFRIDGE, J. L. ....................... 101

Computational Investigations of Least-Squares Type Methods for the Approximate Solution of Boundary

Value. Problems 777

Calculation and Applications of Epstein Zeta Functions ...... 271

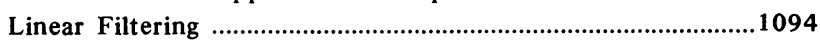

A Class of Hessenberg Matrices with Known Pseudoinverse and Drazin Inverse .......................................................... 615

A Fourier Method for the Numerical Solution of Poisson's

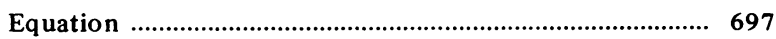

Some Analytic or Asymptotic Confluent Expansions for Functions of Several Variables ............................................1115

On Complex Quadratic Fields with Class-Number Two ........ 289

See: ROSENBERG, IVO G. \& STENGER, FRANK ……..... 390

Gershgorin Theory for the Generalized Eigenvalue Problem

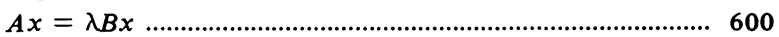

See: HAGER, WILLIAM W. \& STRANG, GILBERT ..............1020

A Combinatorial Theorem in Group Theory ……………….... 303

See: ERDÖS, P., GRAHAM, R. L., RUZSA, I. Z. \& STRAUS, E. G.

Uniform Asymptotic Expansions of the Incomplete Gamma Functions and the Incomplete Beta Function ....................1109

See: JOHNSON, CLAES \& THOMÉE, VIDAR ……………..... 343

See: LUKE, YUDELL L., TING, BING YUAN \& KEMP, MARILYN J. 1083

On Lower and Upper Bounds of the Difference Between the Arithmetic and the Geometric Mean

Power Sum Method and the Approximative Solution of Algebraic Equations 311

See: RUSSELL, R. D. \& VARAH, J. M. .................................1007

Zeros of $p$-Adic $L$-Functions ..................................................1138

See: BATEMAN, PAUL T., PURDY, GEORGE B. \& WAGSTAFF, SAMUEL S., JR.

A Modified Galerkin Procedure with Hermite Cubics for Hyperbolic Problems

See: DOUGLAS, JIM, JR., DUPONT, TODD \& WAHLBIN, LARS

See: GUPTA, G. K. \& WALLACE, C. S. ................................ 489

Factoring Multivariate Polynomials Over the Integers ............ 935

Diagonalization and Simultaneous Symmetrization of the Gas-Dynamic Matrices 1037

On Small Zeros of Dirichlet $L$-Functions 319

Bifurcation in Difference Approximations to Two-Point Boundary Value Problems 746

Differential-Difference Properties of Hypergeometric Polynomials 
Author

WOLFE, JERRY M.

WONG, R.

WUYTACK, LUC

ZAJTA, A. J. \& PANDI-

KOW, W.

ZLÁMAL, MILOŠ
Title

Page

Discrete Rational $L_{p}$ Approximation

On Laplace Transforms Near the Origin

On the Osculatory Rational Interpolation Problem

837

Conversion of Continued Fractions into Power Series

566

Finite Element Multistep Discretizations of Parabolic Boundary Value Problems

350 


\section{SUBJECT CLASSIFICATION SYSTEM \\ FOR INDEX OF REVIEWS}

The following subject classification system is used for the yearly index of reviews. Individual reviews in the quarterly issues are assigned index classification numbers in simplified form.

1.00 Biography and Bibliography (History)

2.00 Selected Topics in Numerical Analysis

2.05 Approximation Theory

2.05.1 Least Squares, Curve Fitting, Harmonic Analysis

2.05.2 Chebyshev (Best) Approximation

2.05.3 Interpolation, Extrapolation

2.05.4 Inverse Interpolation

2.05.5 Rational Approximation

2.05.6 Splines

2.10 Numerical Integration

2.10.1 One-Dimensional

2.10.2 Multi-Dimensional

2.10.3 Monte Carlo

2.15 Numerical Differentiation

2.20 Roots of Equations

2.25 Evaluation of Series

2.30 Continued Fractions

2.35 Iteration Methods, Acceleration Techniques

2.40 Differences, Divided Differences

2.45 Algorithms, General Theory

2.50 Inequalities

2.55 Stability of Computation, Significance Arithmetic

2.60 Complexity of Computation

3.00 Linear Algebra

3.05 Matrices

3.10 Linear Equations

3.10.1 Error Analysis

3.15 Eigenvalues and Eigenvectors

3.15.1 Error Analysis

3.20 Matrix Inversion and Pseudo-Inverses

3.20.1 Error Analysis

3.25 Linear and Nonlinear Programming, Theory of Games 3.25.1 Error Analysis

3.30 Determinants

3.35 Sparse Matrices

4.00 Ordinary Differential Equations

4.05 Initial Value Problems

4.05.1 Analytic Methods

4.05.1.1 Error Analysis

4.05.2 One-Step Methods

4.05.2.1 Error Analysis

4.05.3 Multistep Methods

4.05.3.1 Error Analysis

4.10 Linear Boundary Value and Eigenvalue Problems

4.10.1 Analytic Methods

4.10.1.1 Error Analysis

4.10.2 Initial Value (Trial and Error or Shooting) Methods 4.10.2.1 Error Analysis 
4.10.3 Finite Differen'ce (Nonshooting) Methods

4.10.3.1 Error Analysis

4.10.4 Finite Element Methods

4.10.4.1 Error Analysis

4.15 Nonlinear Boundary Value and Eigenvalue Problems

4.15.1 Analytic Methods

4.15.1.1 Error Analysis

4.15.2 Initial Value (Trial and Error or Shooting) Methods 4.15.2.1 Error Analysis

4.15.3 Finite Difference (Nonshooting) Methods

4.15.3.1 Error Analysis

5.00 Partial Differential Equations

5.05 Initial Value Problems

5.05.1 Analytic Methods

5.05.1.1 Error Analysis

5.05.2 Explicit Difference Methods

5.05.2.1 Error Analysis

5.05.3 Implicit Difference Methods, Iterative Schemes 5.05.3.1 Error Analysis

5.05.4 Finite Element and Other Approximation Methods 5.05.4.1 Error Analysis

5.10 Boundary Value Problems

5.10.1 Analytic Methods 5.10.1.1 Error Analysis

5.10.2 Finite Difference Methods, Iterative Schemes 5.10.2.1 Error Analysis

5.10.3 Finite Element and Other Approximation Methods 5.10.3.1 Error Analysis

5.15 Eigenvalue Problems

5.15.1 Analytic Methods 5.15.1.1 Error Analysis

5.15.2 Finite Difference Methods, Iterative Schemes 5.15.2.1 Error Analysis

5.15.3 Finite Element and Other Approximation Methods 5.15.3.1 Error Analysis

5.20 Mixed Initial and Boundary Value Problems

5.20.1 Analytic Methods 5.20.1.1 Error Analysis

5.20.2 Explicit Difference Methods 5.20.2.1 Error Analysis

5.20.3 Implicit Difference Methods, Iterative Schemes 5.20.3.1 Error Analysis

5.20.4 Finite Element and Other Approximation Methods 5.20.4.1 Error Analysis

6.00 Other Functional Equations
6.05 Difference Equations
6.10 Difference-Differential Equations
6.15 Integral Equations
6.20 Integro-Differential Equations
6.25 Convolution Equations
6.30 Variational Equations
6.35 Abstract Operator Equations
6.40 Stochastic Differential Equations

7.00 Special Functions

7.05 Mathematical Constants, Special Polynomials (Nonorthogonal) and Numbers: Bernoulli, Euler, Stirling, Binomial Coefficients, Factorials

7.10 Elementary Functions, Powers and Roots

7.15 Gamma Functions Psi Functions, Zeta Functions and Related Functions 
7.20 Incomplete Gamma Functions

7.20.1 Exponential Integral, Cosine and Sine Integrals and Related Functions

7.20.2 Error Functions, Fresnel Integrals and Related Functions

7.25 Confluent Hypergeometric Functions

7.25.1 Parabolic Cylinder Functions

7.25.2 Coulomb Wave Functions

7.30 Bessel Functions

7.35 Lommel Functions, Struve Functions, Anger-Weber Functions and Associated Bessel Functions

7.40 Legendre Functions

7.45 Gaussian Hypergeometric Functions

7.50 Orthogonal Polynomials and Functions

7.55 Expansions in Series of Orthogonal Polynomials, Bessel Functions, Other Functions

7.60 Elliptical Integrals and Functions, Weierstrass Elliptic Integrals, Related Functions

7.65 Mathieu Functions

7.70 Spheroidal Wave Functions, Other Wave Functions

7.75 Generalized Hypergeometric Functions of a Single Variable

7.80 Generalized Hypergeometric Functions of More Than One Variable

7.85 Basic Hypergeometric Functions

7.90 Integral Transforms

7.95 Numerical Tables of Integrals and Transforms

7.100 Numerical Tables of Infinite Series

7.105 Handbooks of Mathematical Tables and Formulae

8.00 Probability and Statistics

8.05 Random Numbers

8.05.1 Tables

8.10 Monte Carlo, Markov Chains

8.15 Multivariate Analysis

8.20 Regression Analysis

8.25 Analysis of Variance

8.30 Time Series Analysis

8.35 Nonparametric Analysis

8.40 Sequential Analysis

8.45 Classical Statistics

8.50 Handbooks of Tables and Formulae

9.00 Number Theory

9.05 Mersenne, Fermat, Perfect and Related Numbers

9.10 Number Theoretic Functions and Tables

9.15 Binomial Congruences, Primitive Roots, Residues, etc.

9.20 Primes and Their Distribution

9.25 Factorization

9.30 Forms and Diophantine Equations

9.35 Continued Fractions and Diophantine Approximation

9.40 Normal Numbers and Distribution of Digits

9.45 Modular Computation

10.00 Algebra and Combinatorial Theory

10.05 Groups, Rings, Fields, Algebras

10.10 Finite Fields

10.15 Irreducible Polynomials

10.20 Arrays, Latin Squares

10.25 Projective Planes, Block Designs, Difference Sets

10.30 Permutations, Combinatorial Identities

10.35 Graph Theory

11.00 Geometry

11.05 Coordinate Conversion Tables

11.10 Polyhedra and Polytopes

11.15 Graph Theory 
12.00 Computers and Other Aids to Computation

12.05 Digital Computers

12.05.1 Coding, Programming and Software

12.05.2 Design and Hardware

12.05.3 Computer Aided Instruction

12.10 Analog Computers

12.10.1 Design and Hardware

12.15 Digital-Analog (Hybrid) Computers

12.15.1 Coding, Programming and Software

12.15.2 Design and Hardware

12.20 Mechanical Aids to Computation-Slide Rules, Desk Calculators, Others

12.25 Nomographs

13.00 Applications

13.05 Physical and Chemical Sciences

13.05.1 Weights and Measures Tables

13.10 Astronomy, Astrophysics

13.10.1 Navigation Tables

13.15 Engineering Sciences

13.15.1 Engineering Tables

13.20 Earth Sciences, Atmospheric Sciences, Fluid Dynamics

13.20.1 Triangulation and Geodetic Tables

13.25 Biology and the Behavioral Sciences

13.30 Economics and the Social Sciences

13.35 Information Theory, Automata, Logic Control Theory, Dynamic Programming, Cybernetics

13.40 Management Problems, Data Analysis and Processing

13.45 Actuarial Science

13.45.1 Actuarial and Financial Tables

13.50 Humanities, Linguistics

13.55 Logic

14.00 Miscellaneous

14.05 Mathematical Research, Mathematical Education 


\section{INDEX OF REVIEWS BY AUTHOR OF WORK REVIEWED}

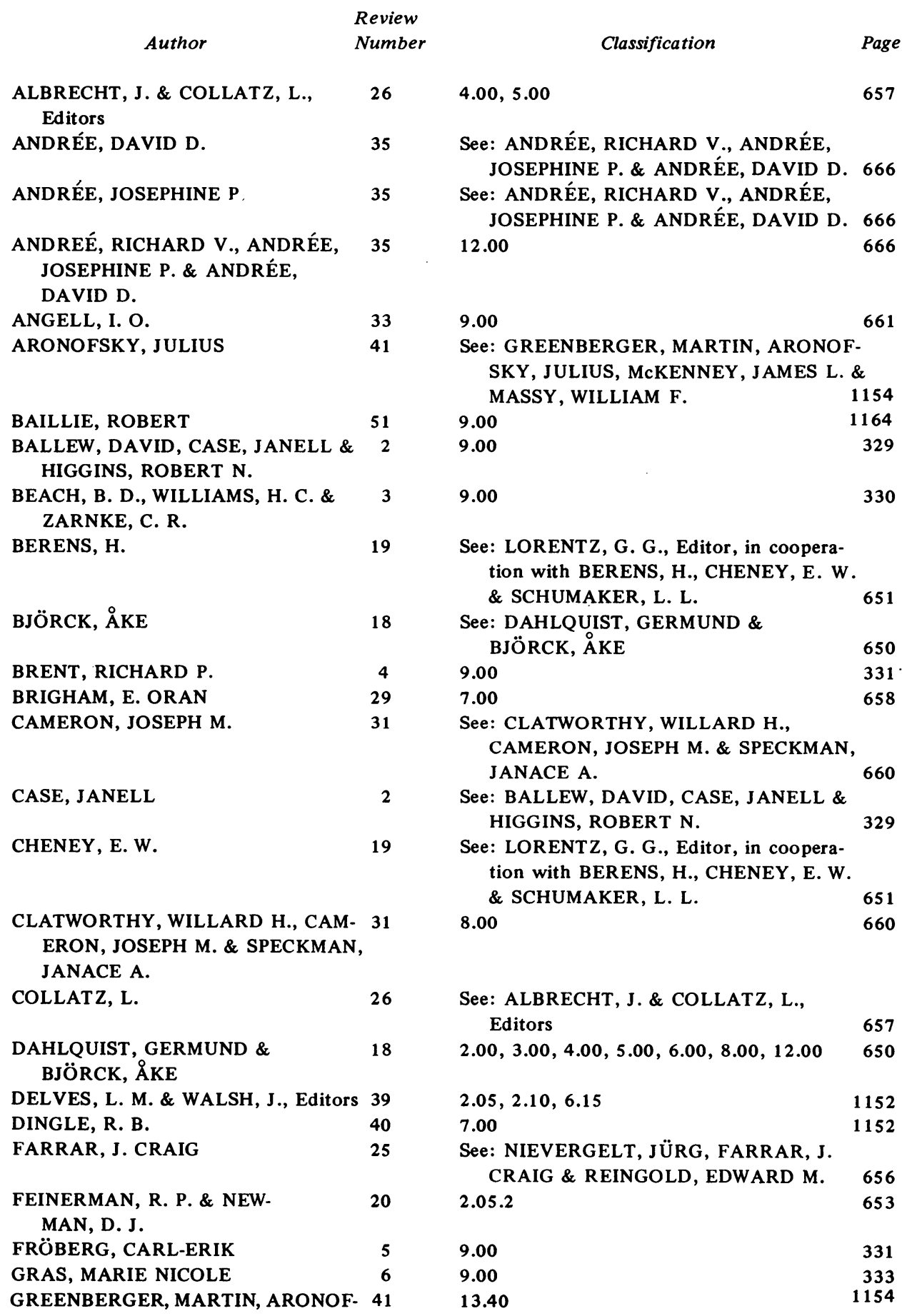


$\begin{array}{ll}\text { Author } & \text { Review } \\ \text { Number }\end{array}$

HAGIS, PETER, JR. \& MCDANIEL, 38 WAYNE L.

HAMMING, R. W.

HARTER, H. L.

HIGGINS, ROBERT N.

HOUSEHOLDER, ALSTON S.

JOHNSON, WELLS

KIRCH, ALLAN M.

KULIK, J. P.

KURODA, SIGEKATU

LAKEIN, RICHARD B.

LIEBERSTEIN, H. MELVIN

LORENTZ, G. G., Editor, in coopera-

tion with BERENS, H., CHENEY,

E. W. \& SCHUMAKER, L. L.

LOWE, BARRY

MCDANIEL, WAYNE L.

MCKENNEY, JAMES L.

MASSY, WILLIAM F.

MURRAY, W., Editor

NEWMAN, D. J.

NEWMAN, MORRIS

NIEVERGELT, JÜRG, FARRAR, J. 25

CRAIG \& REINGOLD, EDWARD M.

OLVER, F. W.

OLVER, F. W.

O'MALLEY, ROBERT E., JR.

OWEN, D. B.

REINGOLD, EDWARD M.

ROBINSON, J.

ROMAN, IRWIN

SALZER, HERBERT E.

SCHAEFER, MARVIN

SCHUMAKER, L. L.

SHANKS, DANIEL

SHANKS, DANIEL \& WRENCH, JOHN W., JR.

SPECKMAN, JANACE A.

\begin{tabular}{lr}
\multicolumn{1}{c}{ Classifications } & Page \\
9.00 & \\
& 968 \\
$2.00,3.00,4.00$ & 648 \\
See: THE INSTITUTE OF MATHEMATI- & \\
CAL STATISTICS, Editors and HARTER, \\
H. L. \& OWEN, D. B., Coeditors \\
See: BALLEW, DAVID, CASE, JANELL \& \\
HIGGINS, ROBERT N. \\
$2.06,3.00$ & 329 \\
9.00 & 1155 \\
9.00 & 334 \\
9.00 & 1155 \\
9.00 & 335 \\
9.00 & 335 \\
13.25 & 336 \\
2.05 & 668 \\
& 651
\end{tabular}

See: WILLIAMS, KENNETH S. \& LOWE, BARRY

See: HAGIS, PETER, JR.\& MCDANIEL, WAYNE $L$.

968

See: GREENBERGER, MARTIN, ARONOFSKY, JULIUS, MCKENNEY, JAMES

L. \& MASSY, WILLIAM F.

1154

See: GREENBERGER, MARTIN, ARONOFSKY, JULIUS, MCKENNEY, JAMES L. \& MASSY, WILLIAM F. 1154

$2.35 \quad 655$

See: FEINERMAN, R. P. \& NEWMAN, D. J.

653

$9.00 \quad 336$

$3.00,7.00,8.00,10.00 \quad 656$

$2.25,4.00,7.00$

$2.25,4.00,7.00$

$4.00 \quad 1156$

See: THE INSTITUTE OF MATHEMATI-

CAL STATISTICS, Editors and HARTER,

H. L. \& OWEN, D. B., Coeditors 661

See: NIEVERGELT, JÜRG, FARRAR, J.

CRAIG \& REINGOLD, EDWARD M. 656

$\begin{array}{ll}5.10 .3 & 1157\end{array}$

$7.00 \quad 658$

$2.05,7.00 \quad 653$

12.05.1 1158

See: LORENTZ, G. G., Editor, in cooperation with BERENS, H., CHENEY, E. W. \& SCHUMAKER, L. L. 651

$9.00 \quad 337$

\begin{tabular}{l}
$7.00 \quad 329$ \\
\hline
\end{tabular}

See: CLATWORTHY, WILLARD H., CAMERON, JOSEPH M. \& SPECKMAN, JAN-

ACE A. $\quad 660$

$7.00 \quad 659$

$9.00 \quad 338$ 


\begin{tabular}{|c|c|c|c|}
\hline & Review & & \\
\hline Author & Numbẹr & Classifications & Page \\
\hline TABAKOVA, E. D. & 34 & 9.00 & 665 \\
\hline TEMAN, ROGER & 27 & 5.00 & 657 \\
\hline TEMPLEMAN, ANDREW B., Editor & 47 & 13.15 & 1160 \\
\hline $\begin{array}{l}\text { THE INSTITUTE OF MATHEMATI- } \\
\text { CAL STATISTICS, Editors and } \\
\text { HARTER, H. L. \& OWEN, D. B., } \\
\text { Coeditors. }\end{array}$ & 32 & 8.00 & 661 \\
\hline THE OPEN UNIVERSITY & 17 & $2.00,3.00,4.00$ & 649 \\
\hline WALSH, J. & 39 & See: DELVES, L. M. \& WALSH, J., Editors & 1152 \\
\hline WEINGARTEN, FREDERICK W. & 36 & 12.00 & 667 \\
\hline WEINTRAUB, SOL & 48 & 9.20 & 1161 \\
\hline WILLIAMS, H. C. & 3 & $\begin{array}{l}\text { See: BEACH, B. D., WILLIAMS, H. C. \& } \\
\text { ZARNKE, C. R. }\end{array}$ & 330 \\
\hline WILLIAMS, H. C. \& ZARNKE, C. R. & 14 & 9.00 & 339 \\
\hline $\begin{array}{l}\text { WILLIAMS, KENNETH S. \& LOWE, } \\
\text { BARRY }\end{array}$ & 15 & 9.00 & 339 \\
\hline WILLOUGHBY, RALPH A., Editor & 49 & 4.00 & 1161 \\
\hline WRENCH, JOHN W., JR. & 1 & See: SHANKS, DANIEL \& WRENCH, JOHI & \\
\hline & & W., JR. & 329 \\
\hline YATES, SAMUEL & 50 & $9.00,9.20$ & 1162 \\
\hline ZARNKE, C. R. & 3 & $\begin{array}{l}\text { See: BEACH, B. D., WILLIAMS, H. C. \& } \\
\text { ZARNKE, C. R. }\end{array}$ & 330 \\
\hline ZARNKE, C. R. & 14 & See: WILLIAMS, H. C. \& ZARNKE, C. R. & 339 \\
\hline
\end{tabular}

\section{INDEX OF REVIEWS BY SUBJECT OF WORK REVIEWED}

$\begin{array}{ll}\text { Author } & \begin{array}{l}\text { Review } \\ \text { Number }\end{array}\end{array}$

2.00 Selected Topics in Numerical Analysis

DAHLQUIST, GERMUND \& 18

BJÖRCK, АKE

HAMMING, R. W.

HOUSEHOLDER, ALSTON S.

THE OPEN UNIVERSITY

2.05 Approximation Theory

DELVES, L. M. \& WALSH, J., Editors 39

LORENT Z, G. G., Editor, in coopera- 19

tion with BERENS, H., CHENEY,

E. W. \& SCHUMAKER, L. L.

SALZER, HERBERT E.

2.05.2 Chebyshev (Best) Approximation

FEINERMAN, R. P. \& NEWMAN, D. J. 20

2.10. Numerical Integration

DELVES, L. M. \& WALSH, J., Editors 39

2.25 Evaluation of Series

OLVER, F. W

OLVER, F. W.

22

2.35 Iteration Methods, Acceleration Techniques

MURRAY, W., Editor

3.00 Linear Algebra

DAHLQUIST, GERMUND \&

BJÖRCK, ÅKE
23

24

Numerical Methods $\quad 650$

Numerical Methods for Scientists and Engineers

Principles of Numerical Analysis $\quad 1155$

Course Books

649

Numerical Solution of Integral Equations 1152

Proceedings of an International Symposium Conducted by the University of Texas and the National Science Foundation, January 22-24, 1973

Laplace Transforms of Osculatory Interpolation Coefficients

Polynomial Approximation

Numerical Solution of Integral Equations

Asymptotics and Special Functions

Introduction to Asymptotics and Special Functions

Numerical Methods for Unconstrained Optimization 


$\begin{array}{lll} & \text { Review } & \\ \text { Nuthor } & \text { Title } & \text { Page }\end{array}$

HAMMING, R.W.

HOUSEHOLDER, ALSTON S. $\quad 42$

NIEVERGELT, JÜRG, FARRAR, J. 25 CRAIG \& REINGOLD,

- EDWARD M.

THE OPEN UNIVERSITY

4.00 Ordinary Differential Equations

ALBRECHT, J. \& COLLATZ, L., $\quad 26$ Editors

DAHLQUIST, GERMUND \& $\quad 18$ BJÖRCK, ̊̊KE

HAMMING, R. W.

OLVER, F. W.

OLVER, F. W.

O'MALLEY, ROBERT E., JR.

THE OPEN UNIVERSITY

WILLOUGHBY, RALPH A., Editor

5.00 Partial Differential Equations

ALBRECHT, J. \& COLLATZ, L., Editors

DAHLQUIST, GERMUND \& BJÖRCK, ÅKE

TEMAN, ROGER

5.10.3 Finite Element and Other

ROBINSON, J.

6.00 Other Functional Equations

DAHLQUIST, GERMUND \& BJÖRCK, А̊KE

6.15 Integral Equations

DELVES, L. M. \& WALSH, J., Editors 39

7.00 Special Functions

BRIGHAM, E. ORAN 29

DINGLE, R. B. 40

NIEVERGELT, JÜRG, FARRAR, J. 25

CRAIG \& REINGOLD, EDWARD M.

OLVER, F. W.

OLVER, F. W.

ROMAN, IRWIN

28

SALZER, HERBERT E.

SHANKS, DANIEL \& WRENCH, JOHN W., JR.,

STROUD, K. A.

8.00 Probability and Statistics

CLATWORTHY, WILLARD H., CAMERON, JOSEPH M. \& SPECKMAN, JANACE A.
Numerical Methods for Scientists and Engineers

Principles of Numerical Analysis

1155

Computer Approaches to Mathematical Problems

Course Books

649

Numerische Methoden bei Differentialgleichungen und mit Funktionalanalytischen Hilfsmit teln

Numerical Methods

Numerical Methods for Scientists and Engineers

Asymptotics and Special Functions

Introduction to Asymptotics and Special

Functions

Introduction to Singular Perturbations $\quad 1156$

Course Books

649

Stiff Differential Systems

1161

Numerische Methoden bei Differentialgleichungen und mit Funktionalanalytischen Hilfsmitteln

Numerical Methods

650

Numerical Analysis

657

Approximation Methods

Integrated Theory of Finite Element Methods

Numerical Methods

650

Numerical Solution of Integral Equations

1152

The Fast Fourier Transform

658

Asymptotic Expansions: Their Derivation and Interpretation

Computer Approaches to Mathematical Problems

Asymptotics and Special Functions

Introduction to Asymptotics and Special Functions

Tables of $N^{3 / 2}$

Laplace Transforms of Osculatory Interpolation Coefficients

Sums of Reciprocals to $1,000,000$

Laplace Transforms, Programmes and Solutions

Tables of Two-Associate-Class Partially Balanced Designs 


Author
DAHLQUIST, GERMUND \&
BJÖRCK, AKE
NIEVERGELT, JÜRG, FARRAR, J.
CRAIG \& REINGOLD,
EDWARD M.
THE INSTITUTE OF MATHEMATI-
CAL STATISTICS, Editors, and
HARTER, H. L. \& OWEN, D. B.,
Coeditors
9.0O Number TheOrY
ANGELL, I. O.
BAILLIE, ROBERT
BALLEW, DAVID, CASE, JANELL \&
HIGGINS, ROBERT N.
BEACH, B. D., WILLIAMS, H. C. \&
ZARNKE, C. R.
BRENT, RICHARD P.
FRÖBERG, CARL-ERIK
GRAS, MARIE NICOLE

HAGIS, PETER, JR. \& McDANIEL, 38 WAYNE $L$.

JOHNSON, WELLS

KIRCH, ALLAN M.

KULIK, J.P.

KURODA, SIGEKATU

LAKEIN, RICHARD B.

NEWMAN, MORRIS

SHANKS, DANIEL

SWIFT, J. D.

TABAKOVA, E. D.

WILLIAMS, KENNETH S. \& LOWE, 15 BARRY

WILLIAMS, H. C. \& ZARNKE, C. R. 14 YATES, SAMUEL 50

9.20 Primes and Their Distribution WEINTRAUB, SOL

10.00 Algebra and Combinatorial Theory NIEVERGELT, JÜRG, FARRAR, J. 25

CRAIG \& REINGOLD, EDWARD M.

\begin{tabular}{lc}
\multicolumn{1}{c}{ Title } & Page \\
Numerical Methods & 650 \\
$\begin{array}{l}\text { Computer Approaches to Mathematical Prob- } \\
\text { lems }\end{array}$ & 656 \\
$\begin{array}{l}\text { Selected Tables in Mathematical Statistics, } \\
\text { Vol. I }\end{array}$ & 661
\end{tabular}

Table of Complex Cubic Fields $\quad 661$

Data on Artin's Conjecture $\quad 1164$

Table of $\phi(n)=\phi(n+1) \quad 329$

Some Computer Results on Units of Quadratic and Cubic Fields $\quad 330$

Tables Concerning Irregularities in the Distribution of Primes and Twin Primes 331

Kummer's Förmodan 331

Méthodes et Algorithmes pour le Calcul Numérique du Nombre de Classes et des Unités des Extensions Cubiques Cycliques de $Q$

A Proof that Every Odd Perfect Number has a Prime Factor Greater than $100110 \quad 968$

The Irregular Primes to 30000 and Related Tables

Elementary Number Theory: A Computer Approach

Magnus Canon Divisorum ...

Table of Class Numbers, $h(p)$ Greater Than 1, for Fields $Q(\sqrt{ } p), p \equiv 1(\bmod 4) \leqslant$ 2776817

Class Numbers of 5000 Quartic Fields $Q(\sqrt{ } \pi)$

A Table of the Coefficients of the Modular Invariant $j(\tau)$

Table of the Greatest Prime Factor of $N^{2}+1$ for $N=1(1) 185000$

Table of Carmichael Numbers to $10^{9}$

A Numerical Investigation of Kummer Cubic Sums (in Russian)

Table of Solutions $(x, u, v, w)$ of the Diophantine System $16 p=x_{2}^{2}+50 u^{2}+50 v^{2}$ $+125 w^{2}, x w=v^{2}-4 u v-u^{2}, x \equiv 1$ $(\bmod 5)$ for Primes $p<10000, p \equiv 1$ $(\bmod 5)$

A Table of Fundamental Units for Cubic Fields

Prime Period Lengths

Computer Program for Compact Prime List 1161 Prime Period Lengths

1162

Computer Approaches to Mathematical Problems 


$\begin{array}{lll} & \text { Review } & \\ \text { Author } & \text { Number } & \text { Title }\end{array}$

12.00 Computers and Other Aids to Computation

ANDRÉE, RICHARD V., ANDRÉE, $35 \quad$ Computer Programming: Techniques, AnalyJOSEPHINE P. \& ANDRÉE, sis and Mathematics

DAVID D.

$\begin{array}{llll}\text { DAHLQUIST, GERMUND \& } & 18 & \text { Numerical Methods } & 650\end{array}$

BJÖRCK, ÁKE

WEINGARTEN, FREDERICK W. $36 \quad$ Translation of Computer Languages

12.05.1 Coding, Programming and Software

SCHAEFER, MARVIN $46 \quad$ A Mathematical Theory of Global Program

13.15 Engineering Sciences

TEMPLEMAN, ANDREW B., Editor

47 Engineering Optimization. Vol. 1

Optimization

13.25 Biology and the Behavioral Sciences

LIEBERSTEIN, H. MELVIN

$37 \quad$ Mathematical Physiology, Blood Flow and

Electrically Active Cells

13.40 Management Problems, Data Analysis and Processing

GREENBERGER, MARTIN, ARONOF- 41 Networks for Research and Education:

SKY, JULIUS, MCKENNEY, JAMES Sharing Computer and Information

L. \& MASSY, WILLIAM F.

Resources Nationwide

1154

\section{INDEX OF TABLE ERRATA}

No.

Author

520 BRENT, RICHARD P.

515 CAYLEY, ARTHUR

516 ERDÉLYI, A., MAGNUS, W., OBERHETTINGER, F. \&

TRICOMI, F. G.

517 HAYASHI, K.

518 HAYASHI, K.

514 LEHMER, D. N.

519 MAGNUS, W., OBERHETTINGER, F. \& SONI, R. P.
Title

Page

Algorithms for Minimization Without Deriva- 1166 tives

An Elementary Treatise on Elliptic Functions $\mathbf{6 7 0}$

Higher Transcendental Functions, Vol. II

670

Tafeln für die Differenzenrechnung sowie für die Hyperbel-, Besselschen, elliptischen und anderen Funktionen

Tafeln der Besselschen Theta-, Kugel- und anderer Funktionen

670

List of Prime Numbers from 1 to $10,006,721341$

Formulas and Theorems for the Special Functions of Mathematical Physics

\section{INDEX OF CORRIGENDA}

Author Title Page

BENKOSKI, S. J. \& “On weird and pdeusoperfect numbers"

ERDÖS, P.

FETTIS, HENRY E. "A stable algorithm for computing the inverse error function in the 'tail-end' region"'

GOLDBERG, M. \&

ABARBANEL, $S$. MICULA, G.

"Stable approximations for hyperbolic systems with moving internal boundary conditions"

"Approximate solution of the differential equation $y "=f(x, y)$ with spline functions" $\quad 673$

SHANKS, DANIEL

"Calculation and applications of Epstein zeta functions" 


\section{INDEX OF ADDENDA}

Author Title

Page

DRESSLER, ROBERT E.

\& PARKER, THOMAS

An addendum to 12,758

675

INDEX OF MICROFICHE SUPPLEMENTS

Author

ESPER, N.

WEINTRAUB, SOL
Title

MOC Issue

"Tables of Reductions of Symmetrized Inner Products ("Inner Plethysms") of Ordinary Irreducible Representations of Symmetric Groups"

October

Computer Program for Compact Prime List
October 